\title{
Use of simulation to optimize the pinhole diameter and mask thickness for an X-ray backscatter imaging system
}

\author{
A.Vella ${ }^{* a}$, Andre Munoz ${ }^{a}$, Dr Matthew J. F. Healy ${ }^{a}$, Dr David Lane ${ }^{a}$, D.Lockley ${ }^{b}$ \\ ${ }^{a}$ Cranfield University, Shrivenham Campus, Watchfield, SN6 8LA, United Kingdom; ${ }^{b}$ Counter \\ Terrorism \& Security Division, Dstl, Fort Halstead, United Kingdom
}

\begin{abstract}
The PENELOPE Monte Carlo simulation code was used to determine the optimum thickness and aperture diameter of a pinhole mask for X-ray backscatter imaging in a security application. The mask material needs to be thick enough to absorb most X-rays, and the pinhole must be wide enough for sufficient field of view whilst narrow enough for sufficient image spatial resolution. The model consisted of a fixed geometry test object, various masks with and without pinholes, and a 1040 x 1340 pixels' area detector inside a lead lined camera housing. The photon energy distribution incident upon masks was flat up to selected energy limits. This artificial source was used to avoid the optimisation being specific to any particular X-ray source technology. The pixelated detector was modelled by digitising the surface area represented by the PENELOPE phase space file and integrating the energies of the photons impacting within each pixel; a MATLAB code was written for this. The image contrast, signal to background ratio, spatial resolution, and collimation effect were calculated at the simulated detector as a function of pinhole diameter and various thicknesses of mask made of tungsten, tungsten/epoxy composite or bismuth alloy. A process of elimination was applied to identify suitable masks for a viable X-ray backscattering security application.
\end{abstract}

Keywords: Monte Carlo simulation, PENELOPE, X-ray backscatter, pinhole, MATLAB

\section{INTRODUCTION}

X-ray imaging by backscatter rather than transmission opens new possibilities in the fields of medical imaging, industrial non-destructive testing and security screening, especially where physical access can be an issue. However, imaging by backscatter requires some form of X-ray optics to attribute photons as being scattered or generated from some region of a target. Transmission radiography avoids optics just by the X-ray source being a small point, this achieves successful transmission images but would still fail to image fluorescence generated widely throughout the target. In this work, a simulation code was used (supported by limited experimentation) to rapidly and cost effectively design and forecast the characteristics of options for X-ray backscatter optics. The Monte Carlo simulation tool PENELOPE [1] was chosen for its versatility and the transparency of its coding and underlying principles.

This work focuses on pinhole imaging systems, although the principles can be applied to coded apertures [2] which have the potential to reduce image acquisition times. For a well shielded camera the only X-rays reaching the detector would be those passing through the aperture of the pinhole, and those penetrating the bulk of the mask or the reduced thickness obliquely through the pinhole edges. An ideal pinhole mask would have a very narrow aperture to give high spatial resolution and would be thick enough to prevent X-rays leaking through the surrounding material. However, thick masks can restrict the field of view and could give rise to partial coding in coded aperture systems [3]. The aim for this work is to propose optimum materials, thicknesses and pinhole diameters for a viable security screening application.

*Corresponding author.

E-mail address: a.vella@cranfield.ac.uk. 


\section{MATERIALS AND METHODS}

\subsection{The imaging system and modelling with PENELOPE}

The illustrative X-ray backscatter imaging system comprises a silicon detector enclosed by lead shielding, a brass rotation stage, and a pinhole mask placed in front of the rotation stage. Both sub-tools of PENELOPE; PENGEOM [4] and PENMAIN [5], were used to design the imaging system and execute the Monte Carlo simulation. The mask is simulated for three different materials, different thicknesses and aperture sizes. Each material was examined at three thicknesses without the pinhole to characterise the leakage through the mask material. Characteristic spectra were generated in PENELOPE as energy probability distributions. This was repeated by adding to each of three thicknesses and each of three materials a pinhole aperture of three different diameters. The simulated 'image' at the silicon detector was then collected as a phase-space file by the main program PENMAIN and was processed using MATLAB [6] to show the detector energy response, and explore the noise, contrast and resolution. The X-ray source and test object are discussed in section 2.3.

\subsection{Designing the camera in PENGEOM}

The model is based on a real detector and camera in use at Cranfield University. The imaging system comprises a Gemstar X-ray detector in a lead lined black aluminium tube. The tube is fixed to an aluminium mount clamped securely to an optical bench. Attached to the front of the camera case is a $100 \mathrm{~mm}$ lead lined aluminium extension tube which supports a brass rotation stage. The inner face of the rotation stage is lined with $4 \mathrm{~mm}$ lead sheet. The pinhole mask is supported at the very front of the camera in an aluminium plate attached to the stage, and the whole system is tightly sealed other than the apertures of the pinhole optic. The experimental camera is shown in figure 1a. The model of the camera replicates the relevant features of the experimental setup, and can be seen in figures 1b-c. It contains the following components

- The pinhole mask, in pink, with a thickness of either $0.5,1,2,3,4,5,6,7,8,9$ and $10 \mathrm{~mm}$ and an aperture diameter of either 1,2 or $3 \mathrm{~mm}$. The mask has a $9 \mathrm{~cm}$ overall diameter.

- An aluminium support disk, in red, $1 \mathrm{~cm}$ thick, $20 \mathrm{~cm}$ external diameter and $9 \mathrm{~cm}$ diameter inner hole;

- The brass rotation stage, in purple, $4.7 \mathrm{~cm}$ thick, $20 \mathrm{~cm}$ wide with a $9 \mathrm{~cm}$ diameter inner hole;

- The silicon detector, in orange, $9.24 \times 12.32 \mathrm{~cm}$, sitting $16.6 \mathrm{~cm}$ behind the pinhole.

- Two $16.6 \mathrm{~cm}$ diameter aluminium cylinders, in green and red, to support the pinhole and detector.

The volume surrounding the detector was filled with air to make the simulation more realistic. Air is represented in yellow/gold in the detector section on figure 1c.

\subsection{Thin wire $\mathrm{X}$-ray emitter test object}

The test object was designed for simultaneous appraisal of the image resolution and field of view from different pinhole masks with minimal computation time. The test object was a long thin metal wire placed $16.6 \mathrm{~cm}$ in front of the pinhole mask to give a magnification of unity. In figure $1 \mathrm{~b}$ it is the thin blue cylinder placed in front of the 'purple' pinhole mask. It was set to be narrow $(0.3 \mathrm{~mm})$ so that broadening reveals resolution deterioration caused by imaging system. The extreme thinness of the object was expected to result in a Gaussian image profile useful to calculate the resolution of the system in terms of its FWHM. It was set to be long $(4.6 \mathrm{~cm})$ where any reduction in its length in the image reveals the collimation effect of the pinhole reducing the Field of View (FOV).

Backscatter imaging is usually performed with a source of X-rays near the camera directed at the target area. X-rays scatter from the target area in all directions, including back to the camera. However, to test the characteristics of various pinhole masks it is computationally more efficient to make the test object the source of X-rays rather than to unnecessarily simulate X-rays that miss the 'wire like' object, radiation interactions inside the wire and follow the propagation of scattered X-rays that would clearly miss the camera. Emission was restricted to a $20^{\circ}$ cone in the forward direction to match the area of the mask and the photons generated had equal probability to be produced everywhere within the source wire. In our case the test object generated photons with a uniform yield across the defined energy 
range so that the transmission function of the pinhole mask is easily apparent and to avoid the findings being specific to pyroelectric or thermionic sources with different anode targets, or filters for hardening the beam.
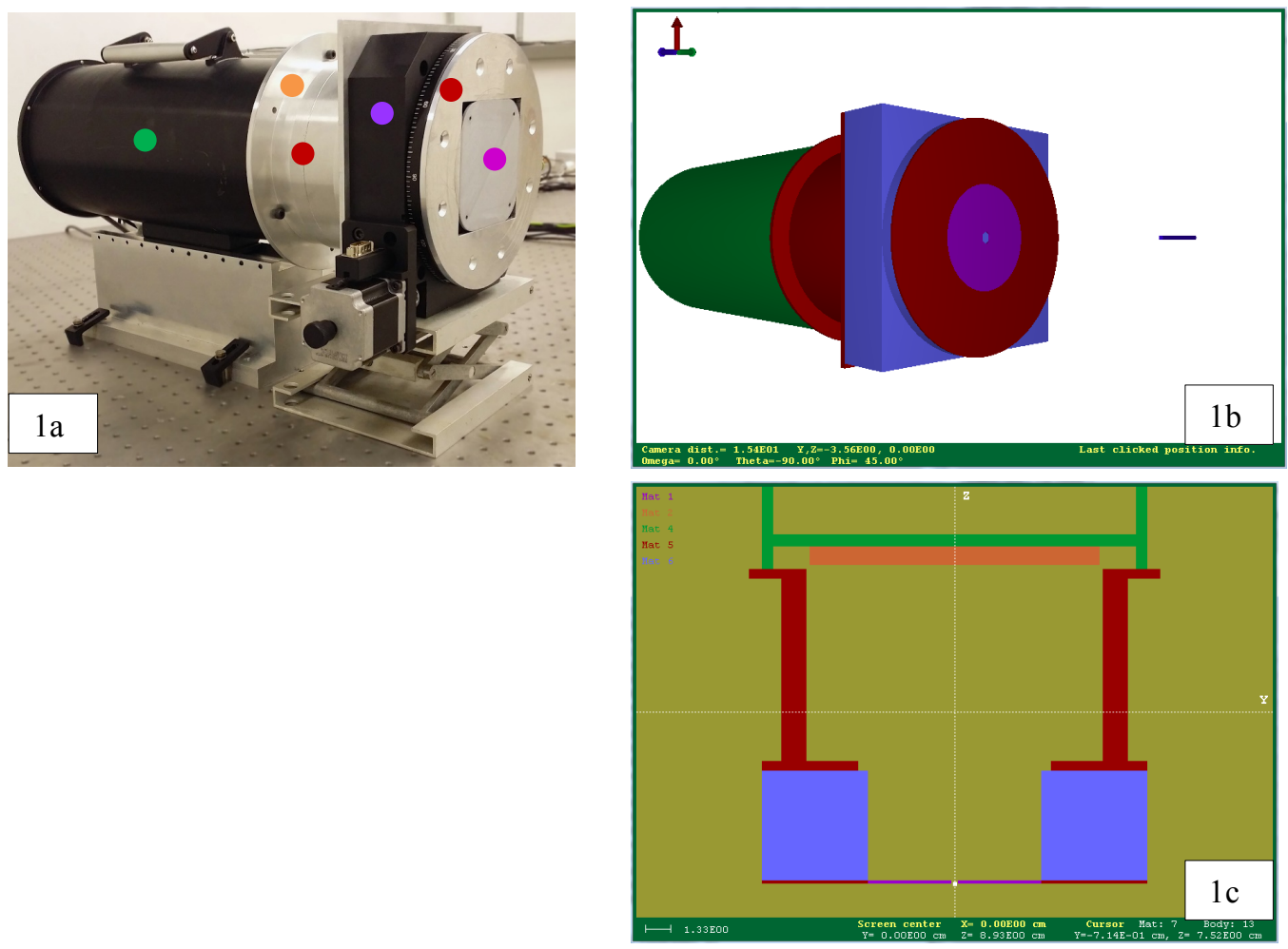

Figure 1. 1a shows the X-Ray camera in the experimental setup, (1b) shows the PENGEOM model in 3D. 1c is a cross section showing the position of the detector inside the shielding. The following components can be seen in $1 \mathrm{~b}$ and $1 \mathrm{c}$ with the PENGEOM colour code:

- Pinhole lens, W, W Ep. Or Bi All.

- Rotation disk, Al

- Rotation stage, Brass

- Extension, $\mathrm{Al}-\mathrm{Pb}$

- External cylindrical envelopment, $\mathrm{Pb}$

- CCD detector, $\mathrm{Si}$

- Wire test object, metal

Simulation permits any mono-energetic, polychromatic, point or extended X-ray source to be modelled whether it is physically feasible yet or not. This allows the user to simulate current real sources, or identify the nature of future sources that might be particularly suitable for imaging or materials characterisation. 


\subsection{Pinholes and mask materials}

The mask materials investigated were solid tungsten metal (which is difficult to machine), tungsten epoxy composite (which can be easily cold cast into complex shapes), and low melting point bismuth alloy (which can be cast but requires heat). The compositions and mass densities are given below

- Tungsten metal (100 wt\%, density $\left.19.6 \mathrm{~g} \mathrm{~cm}^{-3}\right)$

- The tungsten - epoxy composite (W 93.5wt\%, Epoxy A 5.4wt\%, Epoxy B 1.1wt\%, density $9.5 \mathrm{~g} \mathrm{~cm}^{-3}$ )

- The bismuth low melting point alloy (Bi 70.3wt\%, Sn $18.2 \mathrm{wt} \%$, In $11.5 \mathrm{wt} \%$, density $9.6 \mathrm{~g} \mathrm{~cm}^{-3}$ )

Masks between 0.5 and $10 \mathrm{~mm}$ thickness were studied, with circular straight edge pinholes of 0 (no pinhole) 1,2 and $3 \mathrm{~mm}$ diameter. The work of Metzler and Bowsher illustrates chamfered edge pinholes [7].

\section{RESULTS}

\subsection{Energy spectra through the mask materials}

Photon transmission through the material of the mask reduces the contrast and increases noise in images produced using pinhole or coded aperture optics [3]. This section assesses the capacity of nine masks without pinholes to prevent photons reaching the detector from the X-ray emitting wire. The number of photons emitted from the wire was set to $10^{7}$, to achieve acceptable statistics in a relatively short time. The simulated results generated by PENMAIN are recorded in the phase-space file at the model detector, which represents the CCD camera (see in orange in figure 1c). The phase-space file contains diverse useful information including the position, direction and energy of each photon that strikes the model detector. The energy spectra are deduced from this and recorded in a separate file, where the quantity probability/keV refers to the fraction of photons in each $\mathrm{keV}$ width energy bin that reach the detector per $0.1 \mathrm{keV}$ bin. The photon energy spectra from the thin $0.5 \mathrm{~mm}$ thickness masks are shown in figure 2 .

The spectra show several characteristic features caused by their materials. Drops in mask transmission at around 70 and $90 \mathrm{keV}$ are caused by the tungsten and bismuth $\mathrm{K}$ absorption edges, and sharp peaks are caused by fluorescence within the masks. Photons caused by fluorescence are an important feature which extends the ideal mask property from one of preventing transmission of one of incident photons to include not generating secondary photons. Fluorescent photons can be more penetrating than the initiating photon because they can be below the energy of the absorption edge whilst the original photon may be above it. Further, if the application involves characterising the materials of a distant object through a small pinhole then even strong external signals could be obfuscated by weak fluorescence across a large mask, especially if the energy dispersive detector has poor spatial resolution.

\subsection{Introducing a pinhole and adapting the phase-space file to replicate an imaging plate.}

The phase space file (PSF) contains the energy and position data of each photon hitting the detector [1]. This file can be represented as a three dimensional energy-scatter plot, (e.g. figure 3A). However, this does not replicate images in the same way as sensors generally capture images, sensors do not log impacts from individual photons at precise locations but instead measure deposited energy over a time period within digitised spatial regions. To be presented as an image, the raw data of the PSF needs to be processed in a similar way to how the detector actually works; i.e. by integrating the energies of all photons hitting each particular pixel over some exposure period. The integrated energy $\mathrm{I}(\mathrm{E})$ seen in figs $3 \mathrm{~B}-3 \mathrm{C}$ is defined as the sum of the energy of the photon data in the phase space file correlated with the positions and areas of the 1040 x 1390 pixels of the real detector. A MATLAB routine was written to accomplish this mapping and integration. Maps were constructed and show how the integrated energy varies at $x-y$ positions across the surface of the detector (see figure 3B-C) where the 3D map exposes detail but the 2D map more directly indicates the potential of a false colour imager. The images in figure 3 are based on a $4 \mathrm{~mm}$ thick tungsten plate with $2 \mathrm{~mm}$ pinhole equidistant from the test object and detector to give a magnification equal to 1 . 


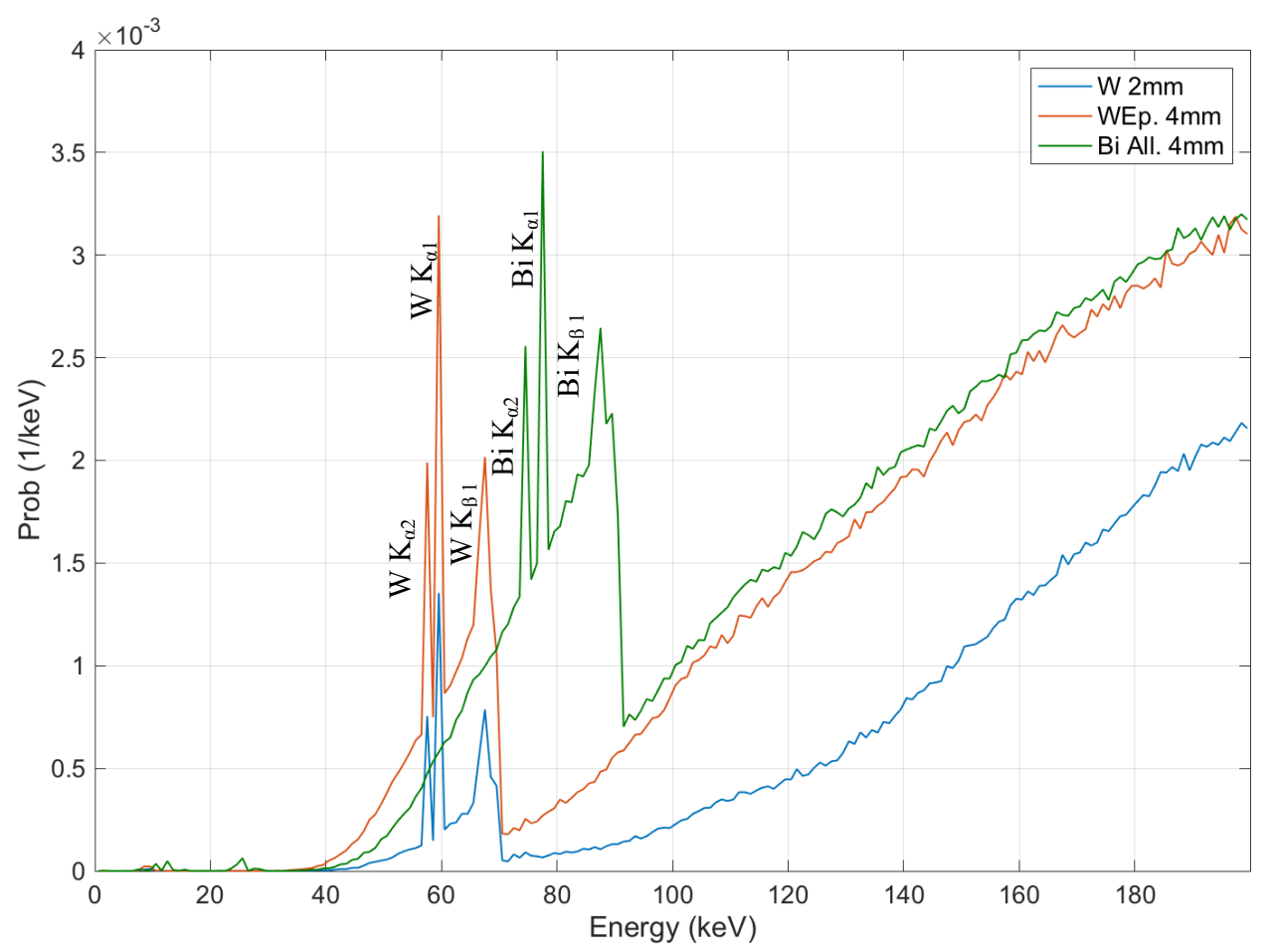

Figure 2. The photon energy-probability spectrum for $0.5 \mathrm{~mm}$ thickness masks for $\mathrm{W}, \mathrm{W}$ Epoxy composite and Bi alloy masks without a pinhole [8].

\subsection{Preliminary appraisal of contrast and field of view with pinhole masks of different thicknesses}

The integrated energy images of the test wire from three mask materials of between 0.5 and $10 \mathrm{~mm}$ thickness were simulated to appraise the influence of the pinhole mask on image characteristics such as contrast and field of view. The pinhole was fixed at $2 \mathrm{~mm}$ and all incident photons are $200 \mathrm{keV}$. Figures 4 to 6 present examples at conditions of an extremely thin mask $(1 \mathrm{~mm})$, two 'typical' working mask thicknesses $(2$ and $4 \mathrm{~mm})$, and an extremely thick mask $(10 \mathrm{~mm})$. The images in figures 4, 5 and 6 compare the effect of different materials whilst increasing their thickness A-D. Because pure tungsten has almost double the density of the alloys and composites, the integrated energy over the whole detector region is lower which is consistent with the energy distribution findings of figure 2 . At $1 \mathrm{~mm}$ thickness there is significant transmission through all mask materials and the images appear noisy. For pure tungsten (fig. 4A), although the wire can be seen it is difficult to distinguish from the background. At the same thickness of tungsten epoxy $(5 \mathrm{~A})$ and bismuth alloy (6A) the wire is almost undistinguishable from the background. At $2 \mathrm{~mm}$, pure tungsten (fig. 4B) prevents enough photons from penetrating the mask body so that photons that travel through the pinhole from the object can be seen as an image above the low background noise across the detector. The epoxy (fig. 5B) and alloy (fig. 6B) allow many photons to penetrate the mask meaning that the object is of similar brightness to the high background. At $4 \mathrm{~mm}$ thickness (fig. 4-5-6C) the mask appears to be sufficiently effective for all the three materials, although for the tungsten epoxy and bismuth alloy the background noise is still present. At 10 $\mathrm{mm}$ the background has been reduced to zero, but the signal from the ends of the wire are clearly not detected indicating collimation (see figure 4-5-6D). The change of colours across the test object in figures 4-6 reveal how the collimation effect increases with thickness. The centre pixel should to a first approximation be unaffected by mask 
material or thickness, however scatter off the inner walls off a pinhole or fluorescence from any mask material will have some influence. These preliminary findings show $2 \mathrm{~mm}$ of pure tungsten and $4 \mathrm{~mm}$ epoxy/alloy appear to be viable thicknesses from which to manufacture pinhole masks or coded apertures. These are the thinnest lightest combinations that reduce background to a subjectively reasonable level. The signal from the three material thicknesses will be explored in the following paragraphs (see section 3.4).

\section{$3 \mathrm{~A}$}

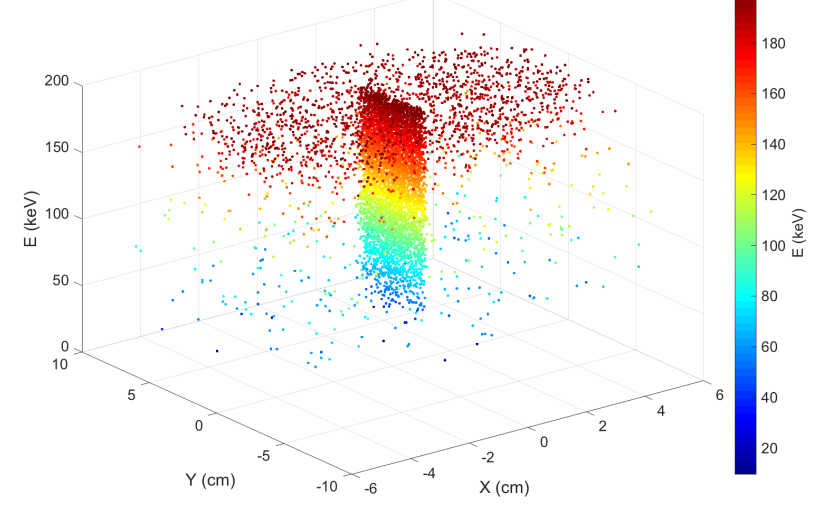

\section{B}


Figure 3. 3A is a scatter plot of the energy of each photon incident on the detector for a $4 \mathrm{~mm}$ thickness $\mathrm{W}$ mask with a $2 \mathrm{~mm}$ diameter aperture (colour scale energy in $\mathrm{keV}$ ). Fig 3B shows the Integrated Energy per pixel calculated by MATLAB presented in three dimensions. Fig 3C shows the same integrated energy per pixel presented in $2 \mathrm{D}$ to reflect the brightness of an image. 


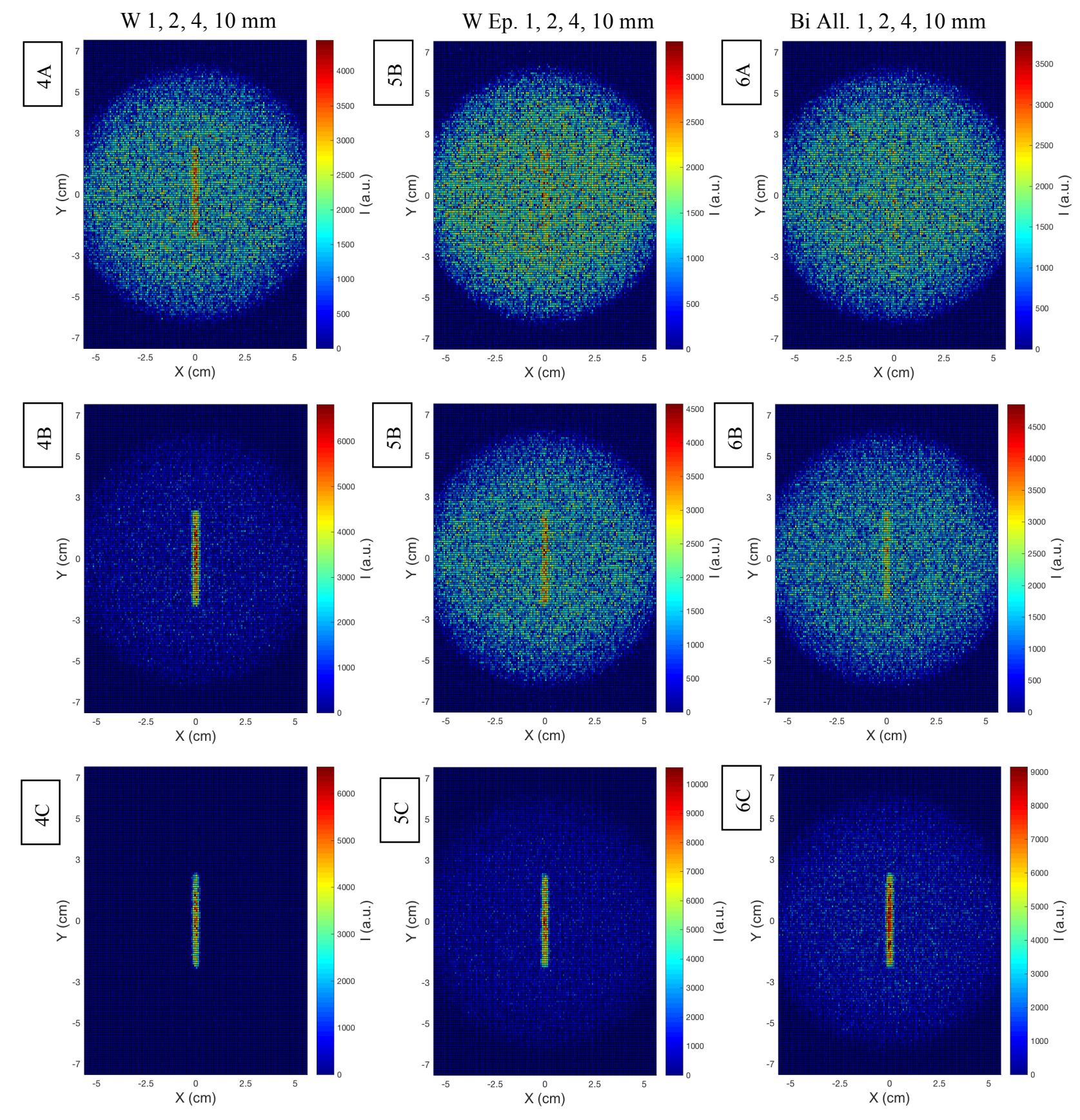



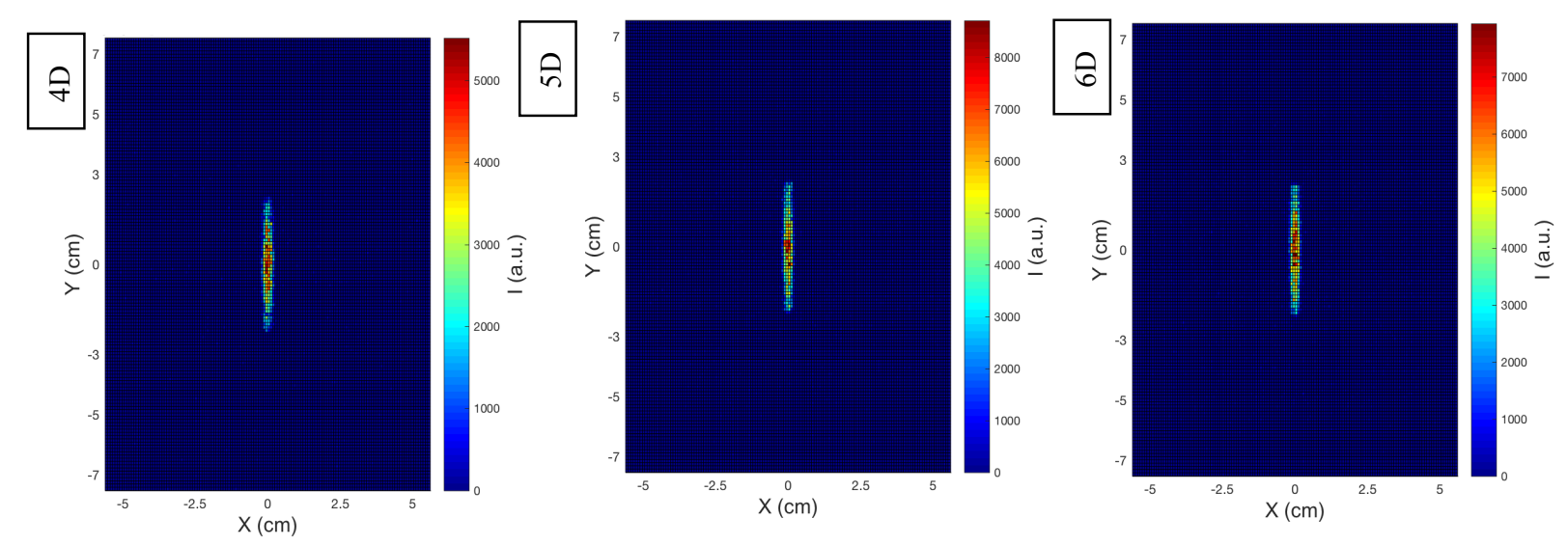

Figure 4. Image of $200 \mathrm{keV}$ photons through pure $\mathrm{W}$ for thickness A $1 \mathrm{~mm}$ (top), B 2mm, C 4mm, D 10mm (bottom)

Figure 5. Image of $200 \mathrm{keV}$ photons through W Epoxy for thickness A $1 \mathrm{~mm}$ (top), B 2mm, C 4mm, D $10 \mathrm{~mm}$ (bottom)

Figure 6. Image of $200 \mathrm{keV}$ photons through Bi alloy for thickness A $1 \mathrm{~mm}$ (top), B 2mm, C 4mm, D 10mm (bottom)

\subsection{Making the evaluation of image characteristics quantitative}

With image characteristics such as noise and resolution, a foundation towards quantification is recognizing where the object image should and should not be in the ideal case. This is a step towards attributing photons as being part of signal of interest or deleterious noise, and establishing the degree of excess or deficit over what should be expected. Figures 4-6 clearly show areas of strong signal and non-zero background. Ideally, the image of the wire should completely occupy the same area as the object $(46 \mathrm{~mm} \times 3 \mathrm{~mm}$, as the object and image are equidistant from the pinhole mask) and there should be no signal detected outside this area. For this study, signal that falls inside this 46x3mmm rectangle will be considered legitimate signal and called the ROI (region of interest) whilst outside it will be considered background.

Figures 7-8-9A (top) show the whole integrated energy image over the detector area for the thinnest viable mask materials identified in the previous section, i.e. $2 \mathrm{~mm}$ thickness for tungsten, $4 \mathrm{~mm}$ for tungsten epoxy and $4 \mathrm{~mm}$ for bismuth alloy. The image is separated into that ideally of just the wire (ROI figs 7-8-9B), and that ideally of just the background (figs 7-8-9C bottom row). The colour scale indicates the integrated energy deposited into each pixel, where red/yellow represents pixels where many (or some high energy) photons reach the detector, and cyan/blue areas represent where few photons (or only low energy photons) hit the detector.

To describe, characterize and compare the image quality parameters of contrast, signal to noise ratio, spatial resolution and field of view signal from the ROI and background regions were evaluated. Calculations were made with MATLAB routines developed in-house and were based on numeric data on the integrated energy, rather than colour as plotted in the images. 
W 2mm, ROI, BKG



$\because$
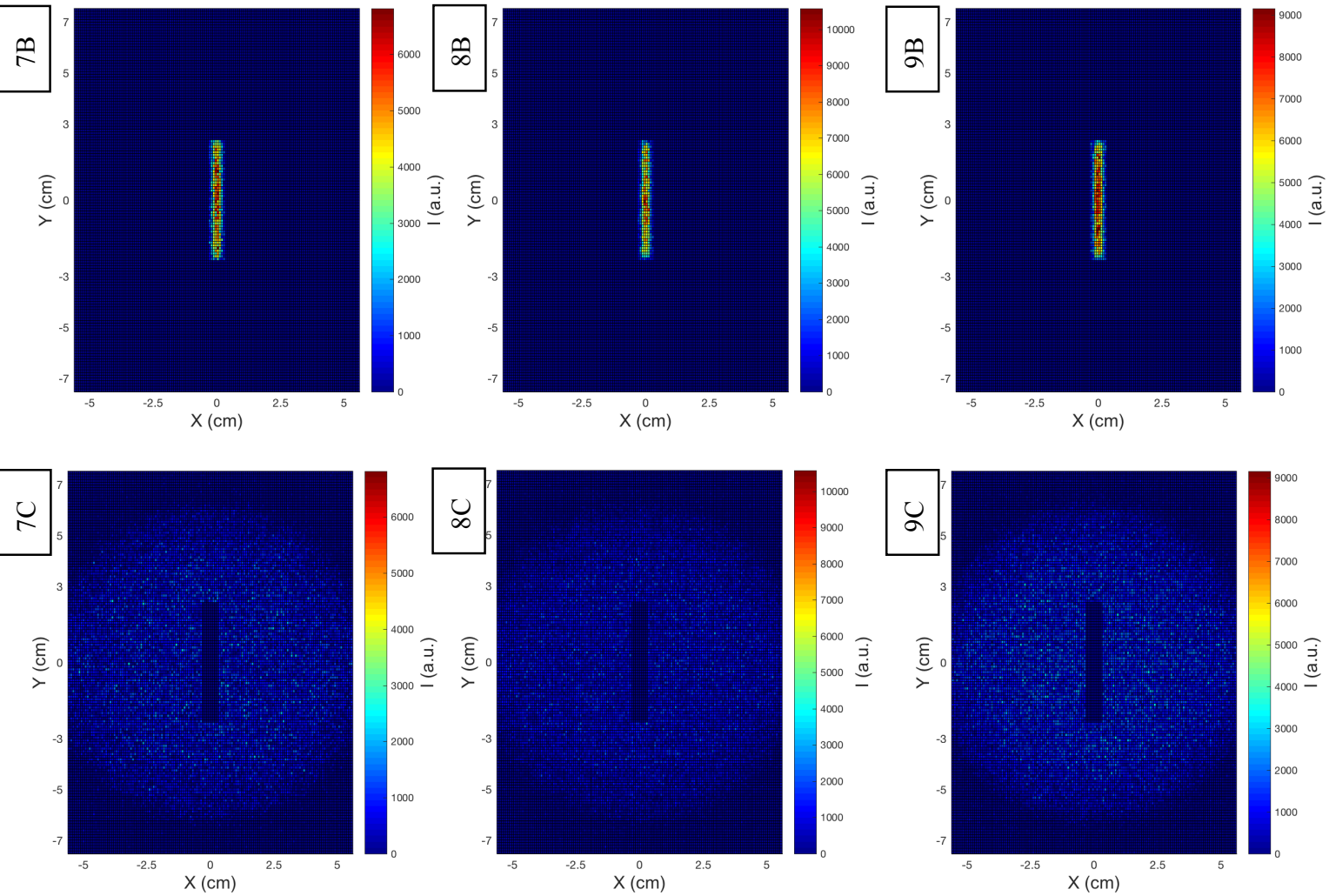

Figure 7. Image obtained from test wire using a $2 \mathrm{~mm}$ aperture in $2 \mathrm{~mm}$ pure tungsten. A represents the whole image, B just the area closely surrounding where the test object signal should geometrically lie, C the background region only.

Figure 8 . As figure 7 but with $4 \mathrm{~mm}$ thickness of $\mathrm{W}$ epoxy. Figure 9 . As figure 7 but with $4 \mathrm{~mm}$ thickness $\mathrm{Bi}$ alloy. 


\subsection{Image contrast}

Contrast is typically defined as the ratio of the signal above background to the average signal [9]. The Michelson contrast or modulated contrast is calculated by

$$
C_{M}=\frac{f_{f}-f_{b}}{f_{f}+f_{b}}
$$

where $f_{\mathrm{f}}$ and $f_{\mathrm{b}}$ represent the total signal and the background, respectively. The Michelson contrast is commonly used where small features are present on a large uniform background, and cannot be more than 1. Michelson contrast is generally considered acceptable when its value is more than 0.1 . (ref). In our case, we calculate the Michelson contrast by considering $f_{f}$ to be the total integrated energy averaged over the whole detector (figures 7-8-9A), and $f_{b}$ as just the background signal (figures 7-8-9C) averaged over the whole pixel grid. This means for the calculation of $f_{f}$, we are not averaging over just the ROI (figures 7-8-9B) but instead over the whole detector area including the background.

The image contrast was calculated for 1, 2 and $3 \mathrm{~mm}$ diameter apertures in all three mask materials for thicknesses ranging from 0.5 to $10 \mathrm{~mm}$. The relative behaviour of these masks for $200 \mathrm{keV}$ incident photons is shown in figures 10A-B-C.

Below $1 \mathrm{~mm}$ thickness all three materials fail to deliver contrast above the 0.1 Michelson criterion. The contrast offered by the pinhole masks increases with both aperture diameter and material thickness. Pure tungsten gives the best contrast in all cases and achieves a contrast exceeding 0.1 at half the thickness of the two other materials, which have similar contrast in all cases. For a $2 \mathrm{~mm}$ diameter aperture, the thinnest masks to exceed the 0.1 contrast criterion are $2 \mathrm{~mm}$ thick solid tungsten and $4 \mathrm{~mm}$ thick epoxy/alloy, which is consistent with the subjective findings of section 3.3. However, for masks of $4 \mathrm{~mm}$ thickness the contrast achieved by solid tungsten is 4 to 6 times greater than that of either $\mathrm{W}$ epoxy or Bi alloy. For $6 \mathrm{~mm}$ pinhole masks pure tungsten achieves 2 to 6 times the contrast of $\mathrm{W}$ epoxy or Bi alloy. The contrast increases with mask thickness until saturation, which likely arises from full photon absorption in the mask. This plateau exists for tungsten exceeding $6 \mathrm{~mm}$ thickness whilst the plateau does not appear to be reached even to $10 \mathrm{~mm}$ thickness for $\mathrm{W}$ epoxy and $\mathrm{Bi}$ alloy. The best contrasts achieved are with the larger diameter pinholes and thick materials. Noting the maximum theoretical contrast is 1.0 , a $3 \mathrm{~mm}$ pinhole and $1 \mathrm{~mm}$ pinhole in $10 \mathrm{~mm}$ of pure tungsten give contrast of 0.95 and 0.7 respectively. However, using high thickness material is unlikely to be practical, especially in cases of narrow pinholes, because of potential side effects such as limited field of view and partial coding with coded apertures.

\subsection{Signal to Noise Ratio (SNR)}

Signal to noise ratio (SNR) is another metric to help evaluate whether an object can be discriminated within an image. The key difference between noise and background is that whilst background may be high it could be completely uniform. An object may be much more easily identified against a uniform background than one that has a large variance. Noise is this variance.

Generally, the acceptable level of SNR is given by the Rose Criterion [10], which states that an object can be distinguishable from background noise if SNR $\geq 5$ and it only occurs when the signal is always positive.

For this study, the signal $\left\langle x_{M}\right\rangle$ is calculated as the value above background of the integrated energy averaged just within the image pixel ROI. The noise $\sigma_{b}$, is the standard deviation of the integrated energy of the background over the whole detector outside the ROI.

$$
S N R=\frac{\left\langle x_{M}\right\rangle}{\sigma_{b}}
$$



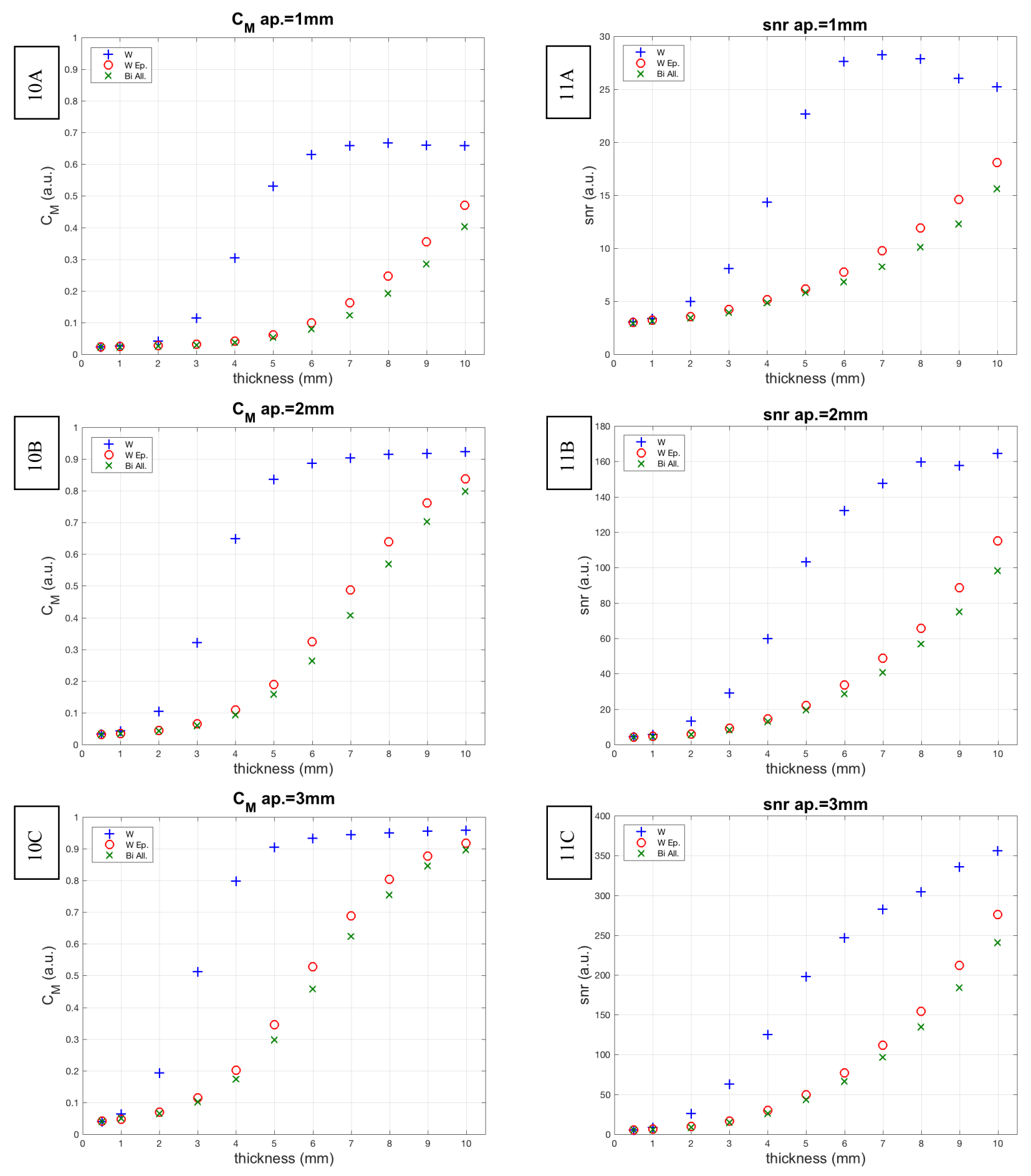

Figure 10. Calculated $\mathrm{C}_{\mathrm{M}}$ for $1 \mathrm{~mm}(\mathrm{~A}), 2 \mathrm{~mm}$ (B) and $3 \mathrm{~mm}$ (C) diameter apertures in masks of varying materials and thicknesses. 
Figure 11. Calculated SNR for $1 \mathrm{~mm}(\mathrm{~A}), 2 \mathrm{~mm}$ (B) and $3 \mathrm{~mm}$ (C) diameter apertures in masks of varying materials and thicknesses.

Figures 11A-B-C show the SNR from various pinhole mask apertures, thicknesses and materials as were simulated in the previous section.

Generally the SNR increases with aperture size and material thickness. However, for a narrow aperture high thicknesses can lead to a collimation effect which reduces the signal in the ROI which is the likely cause of the drop in SNR in Fig 11A. Again, pure tungsten gives better image quality than $\mathrm{W}$ epoxy and Bi alloy for a given thickness, and roughly half the thickness of pure $\mathrm{W}$ is needed to give the same quality as epoxy or alloy. Pure tungsten with a $1 \mathrm{~mm}$ aperture gives an acceptable SNR of 5 when above $2 \mathrm{~mm}$, thickness.

\subsection{Spatial resolution}

Generally, spatial resolution is a metric to quantify the ability of an imaging system to distinguish between two unique objects closely separated in space [11]. The resolution is normally measured by imaging test objects with decreasing separations of parallel lines [12]. Another way to quantify resolution is by defining it in terms of the observed full width at half maximum (FWHM) of a single infinitely narrow object or an infinitely abrupt edge feature. In our case, the FWHM method has been applied across the thin test object where the image intensity in the $\mathrm{x}$ direction of the ROI shows a Gaussian profile. The geometry of the simulation was set to give an image size equal to the $4.6 \mathrm{~cm} \times 0.3 \mathrm{~cm}$ test object. The object image should ideally fully occupy and be confined to the pixels that correspond to the source dimensions. However finite resolution broadens the image beyond these confines. The limits of the ROI were thus set $-2.3 \leq y \leq 2.3$ and $-0.2 \leq x \leq 0.2$ for the smallest aperture, but the $\mathrm{x}$ ROI parameters were raised for larger apertures to ensure image broadening was accommodated. An example image of the test object can be seen in Fig 12A. Although the FWHM along the $\mathrm{x}$ axis used to determine the resolution, it is actually the sum of the integrated energy down the y direction columns is used in order to maximise counting statistics. The column sum as a function of $\mathrm{x}$ is shown in Fig 12B, where a Gaussian fit is applied to determine the FWHM. When the aperture size is $1 \mathrm{~mm}$, the ROI summed histogram presents a lower FWHM, while the signal detected increases in width along $x$ at aperture sizes of $2 \mathrm{~mm}$ and $3 \mathrm{~mm}$, meaning that the lower the FWHM, the higher the resolution is. The graphs on figures 13A-B-C show how the FWHM varies with aperture diameter and mask thickness.

Generally, the resolution improves (FWHM reduces) with reducing pinhole diameter, and apparently reduces with increasing thickness. Thin masks allow large numbers of X-rays hitting the aperture edges obliquely to be transmitted which effectively increases the diameter of the aperture, whilst thick masks only allow X-rays that hit the mask perpendicularly to pass (collimation). Generally, the same spatial resolution achieved by $\mathrm{W}$ epoxy and $\mathrm{Bi}$ alloy can be achieved by half the thickness of pure tungsten. 

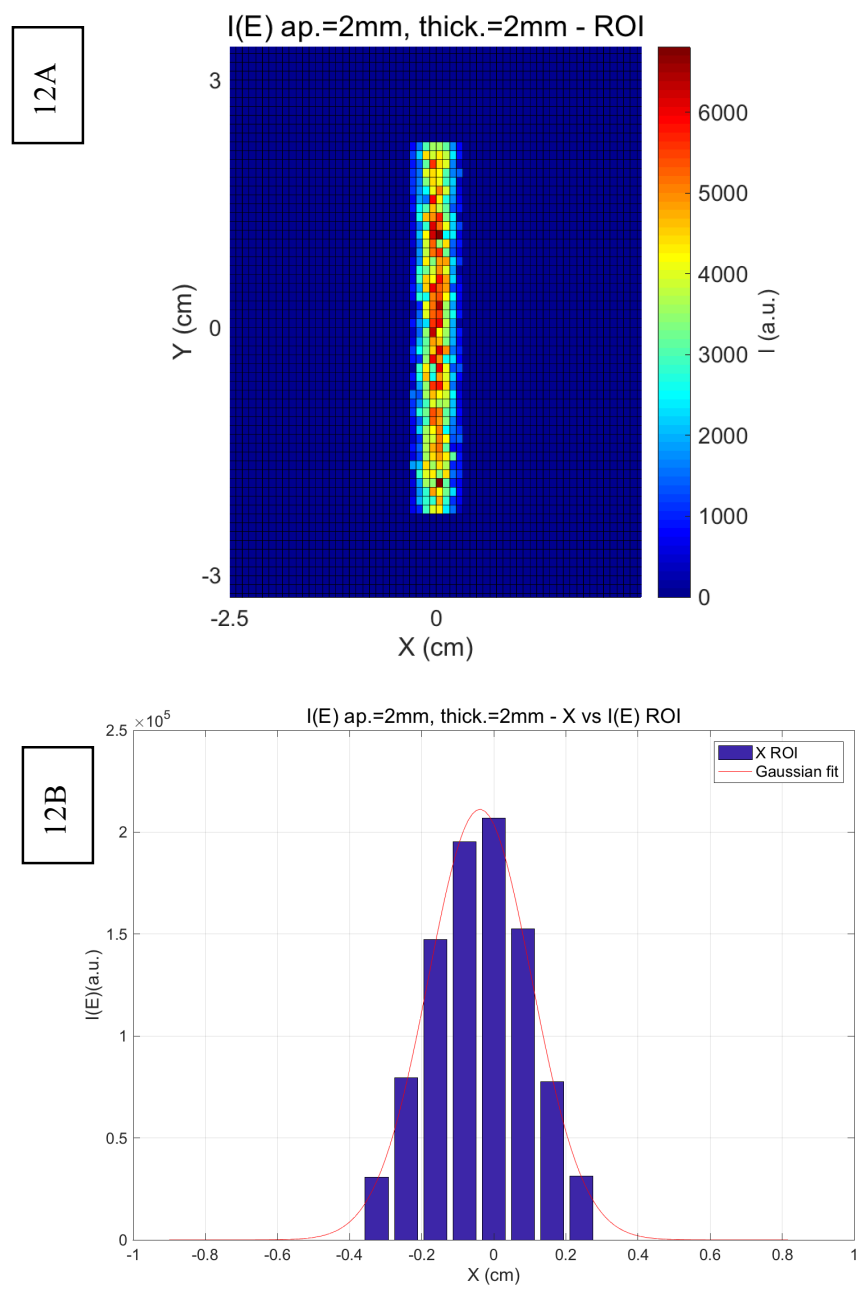

Figure 12. Image of the test object for a $2 \mathrm{~mm}$ thick $2 \mathrm{~mm}$ aperture tungsten pinhole mask (12A) and the summed y-column pixels across the thin $\mathrm{x}$-axis (12B). A Gaussian fit has been plotted on the histogram to calculate the FWHM (red line).

\subsection{The collimation effect}

An ideal pinhole mask would transmit photons through a single locus from across $4 \pi$ steradians. In practice, as the thickness of the mask increases the acceptance angle and the field of view decrease. Simple pinhole images would thus appear cropped at their edges. This effect may be seen in figures 4D, 5D and 6D. Images taken with coded apertures would exhibit partial coding and produce artefacts, even if the nominal FOV is achieved.

The wire test object was designed to be long in order to evaluate the FOV and collimation effect for different pinhole masks. Figure 4D shows that cropping of the wire's image is not abrupt, instead the signal weakens near the ends of the object image. A Gaussian fit along the y axis of the wire's image was used as a simple reproducible measure of its apparent length. Figure 14 shows the collimation effect via the apparent length of the $4.6 \mathrm{~cm}$ test object, as a function of mask material and thickness. Figure 15 shows the effect as function of aperture size, but as the image plate is $16.6 \mathrm{~cm}$ from the pinhole then FOV can be expressed in terms of Angle of View (AOV) for 
general use in other applications. For thin masks with wide apertures the Gaussian fit can extend a little beyond the true length of the wire for reasons including degraded spatial resolution and that the fit is just a convenient simple quantitative measure approximating the true profile. The collimation effect is observed most strongly for thick pure tungsten masks with narrow apertures. Small apertures give a much greater FOV sensitivity to changes in mask thickness, and similarly thick masks give a much greater sensitivity to the AOV. The data presented is based on a test object centred in the field of view, the consequences could be more extreme for objects at the edge of a field of view which are already offset from the axis of a standard cylindrical pinhole. Evaluating the consequences of partial coding across a wide area image taken at short range, arising from collimation in a coded aperture mask is not trivial.

\section{DISCUSSION}

PENELOPE is a useful predictive simulation tool to assist in the design of systems involving X-ray interactions and transport. It has been used to predict the best pinhole mask parameters for a generic X-ray backscatter imaging application in security. However, key characteristics of contrast and signal to noise ratio are dependent on the energy of the X-rays. All X-ray photons incident on the masks in this broadly illustrative scenario are set to $200 \mathrm{keV}$, which deliberately (for reason of penetration into test objects) lies at the higher energy end of small X-ray generators. Also, the majority of current X-ray generators produce most of their X-rays significantly below their maximum energy. The optimum pinhole will likely be different for other X-ray sources. Secondly the Signal to Noise Ratio parameter in particular is dependent on counting statistics and this scenario is based on an overall photon flux that was practical to simulate. In practice the SNR could be better if a longer X-ray exposure was practical; or much worse if the efficiency of the image plate is poor. In applying this work to other situations, the SNR may not be the most reliable parameter on which to choose or eliminate a pinhole mask for applications based on different sources and detectors. This work gives some guidance on viable pinhole masks and also demonstrates a process for designing a convenient test object through applying a simulation tool for modifying the output to match existing pixelated detectors. It also outlines how image characteristics can be simply and reproducibly quantified.

It is possible that complex Multi criteria decision making tools, such as AHP [13] or SMART [14]could be applied in cases where it may be beneficial to balance imaging criterion in a sophisticated way.

\section{CONCLUSIONS}

The PENELOPE Monte Carlo simulation suite has been applied to design, test and optimise the optics of an X-ray backscatter imaging system. PENELOPE has proven to be a versatile tool to model our experimental imaging system and to create a virtual test object with characteristics more suited to design and evaluation than could be achieved experimentally.

A long thin X-ray emitting test wire was shown to be an efficient means to simultaneously characterise a range of parameters such as resolution, field of view, contrast and noise. The creation of MATLAB code to convert PENELOPE's phase space file into the spatial 'brightness' across a typical pixelated imaging plate, enhancing the connection from simulation to existing imaging technology. This allowed simulated images to be collected from a systematically changing set of parameters for pinhole masks, this involved varying the thickness and pinhole aperture diameter of tungsten, tungsten epoxy resin and bismuth alloy. Methods were identified and developed to quantifiably evaluate the image characteristics of contrast, signal to noise ratio, spatial resolution and field of view. For any aperture size, Tungsten epoxy fails to give a sufficiently high contrast and signal to noise ratio until $4 \mathrm{~mm}$ of material is used, whereas pure tungsten can achieve this at $2 \mathrm{~mm}$ thickness. To reduce the background and noise in the presence of a small signal the thickness of pure tungsten must exceed $3 \mathrm{~mm}$ whereas for larger apertures the thickness may be $2 \mathrm{~mm}$. Similarly, for $\mathrm{W}$ epoxy masks, sufficiently low background and high signal to noise ratio can be achieved at $3 \mathrm{~mm}$ thickness for $3 \mathrm{~mm}$ apertures, whereas the thickness must exceed $4 \mathrm{~mm}$ for $2 \mathrm{~mm}$ apertures. Although all the combinations mentioned above allow acceptable field of view, no $3 \mathrm{~mm}$ aperture has acceptable 


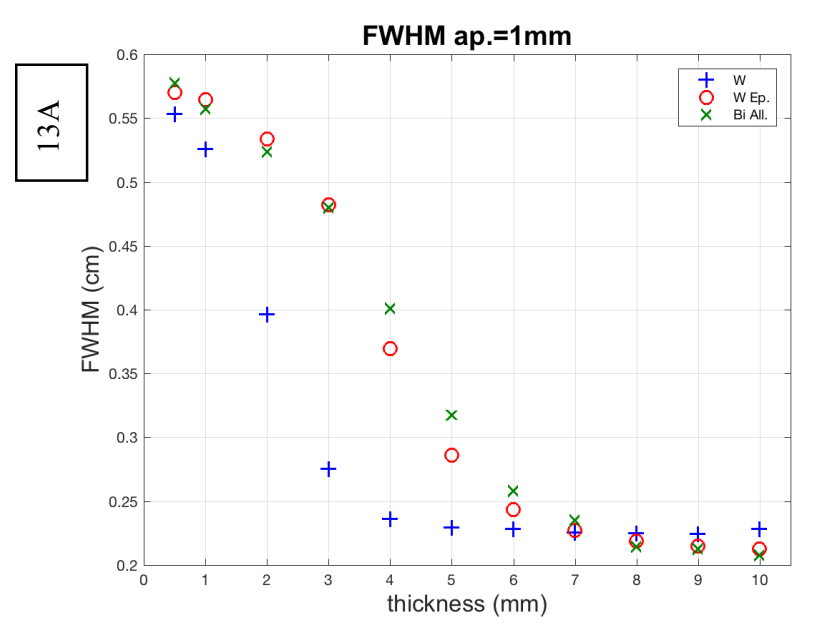

resolution. For the best obtainable resolution, a $1 \mathrm{~mm}$ aperture must be used where only pure tungsten gives both sufficiently high contrast and low noise. The thickness of W Epoxy required to match the background and noise characteristics of $3 \mathrm{~mm}$ thick tungsten is $7 \mathrm{~mm}$, but this would give an unacceptable field of view. Pure tungsten is the most versatile material tested and allows small pinholes to be used in thin masks.
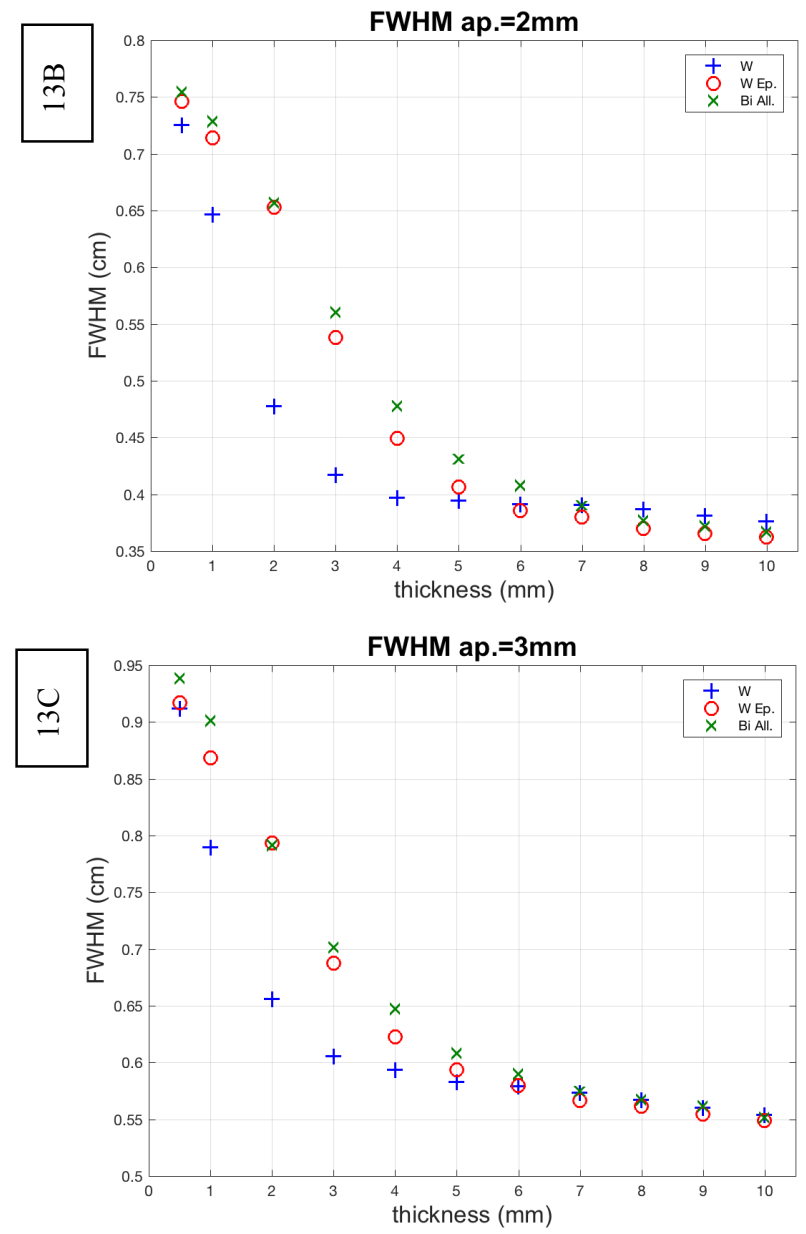

Fig 13. The image spatial resolution available for pinhole masks of different apertures, thicknesses and materials. 

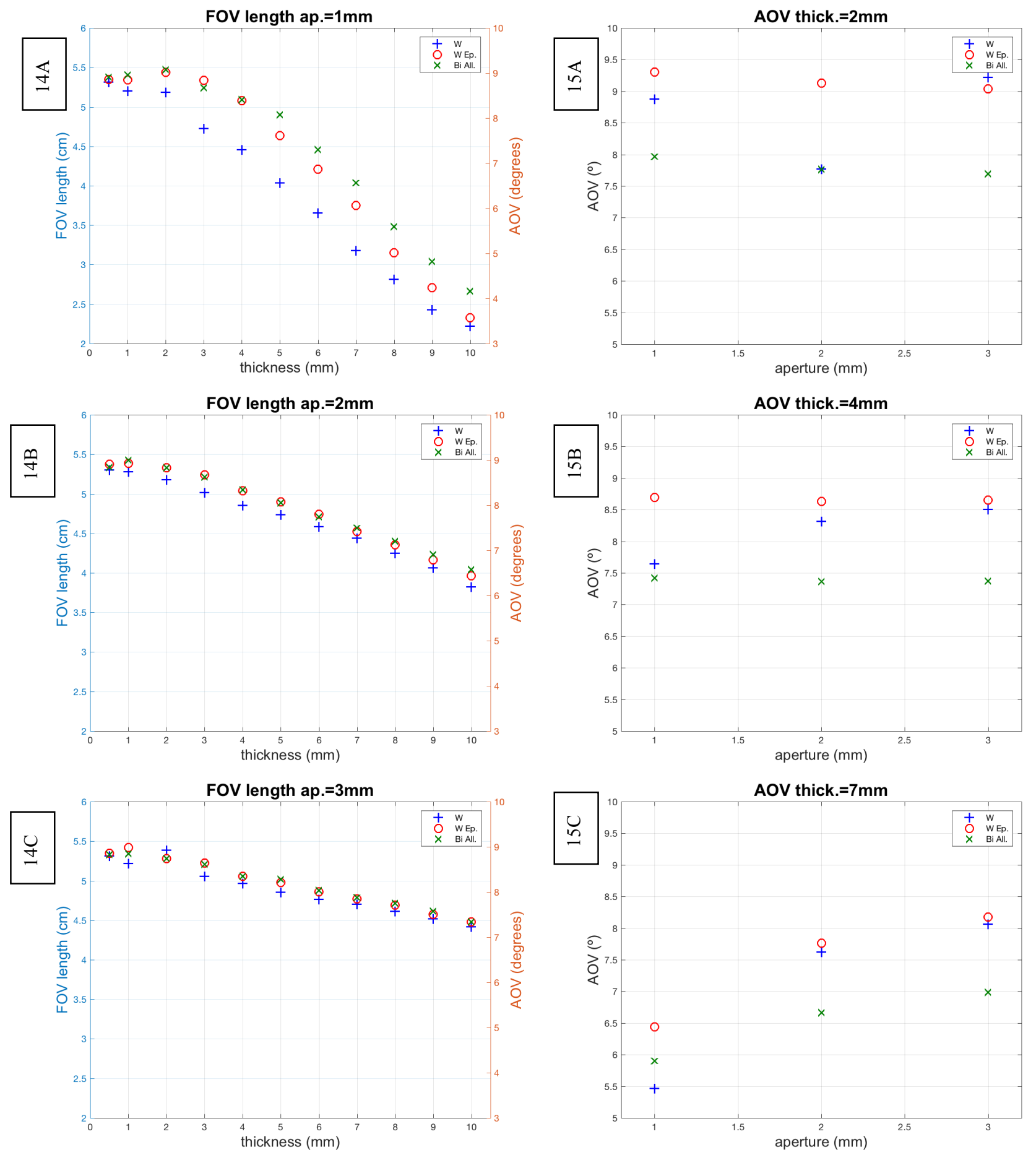

Figure 14A-B-C. The Field of View in terms of the apparent length (left axis) of a $4.6 \mathrm{~cm}$ test object as a function of pinhole mask parameters.

Figure 15A-B-C. The Field of View in terms of angle as a function of pinhole mask parameters. 


\section{ACKNOWLEDGEMENT}

The authors would like to thank Dstl for the financial support for this work.

\section{REFERENCES}

[1] F. Salvat, "PENELOPE-2014: A code system for Monte Carlo simulation of electron and photon transport," OECD/NEA Data Bank, Issy-les-Moulineaux, France, 2015.

[2] E. E. Fenimore, "Coded aperture imaging: predicted performance of uniformly redundant arrays," Applied Optics, vol. 17, no. 22, p. 3563, 1978.

[3] R. Accorsi, F. Gasparini and R. C. Lanza, "A Coded Aperture for High-Resolution Nuclear Medicine Planar Imaging With a Conventional Anger Camera: Experimental Results," IEEE Transactions on Nuclear Science, vol. 48 , no. $6,2001$.

[4] J. Almansa, F. Salvat-Pujol, G. Diaz-Londona, A. Carnicer, A. M. Lallena and F. Salvat, "PENGEOM - A general purpose geometry package for Monte Carlo simulation of radiation transport in complex material structures," Computer Physics Communications (in press), 2015.

[5] F. Salvat and J. M. Fernaddez-Varea, "Overview of physical interaction models for photon and electron transport used in Monte Carlo codes," vol. 46, pp. S122-S138, 2009.

[6] The MathWorks Inc., "MATLAB and Statistics Toolbox Release 2016a," Natick, 2016.

[7] S. D. Metzler and J. E. Bowsher, "Analytic Determination of Pinhole Collimator Sensitivity With Penetration," IEEE Transaction on Medical Imaging, vol. 20, no. 8, 2001.

[8] NIST, "XCOM: Photon Cross Sections Database," 16 April 2016. [Online]. Available: http://www.nist.gov/pml/data/xcom/index.cfm.

[9] A. Michelson, Studies in Optics, Chicago: U. of Chicago Press, 1927.

[10] H. Rose, Adv. Electron., vol. 1, p. 131, 1948.

[11] K. Rossmann, "Point spread-function, line spread-function, and modulation transfer function. Tools for the study of imaging systems," Radiology, pp. 257-272, 1969.

[12] D. R. Dance, S. Christofides, A. D. A. Maidment, I. D. McLean and K. H. Ng, Diagnostic Radiology Physics A handbook for teachers and students, Vienna: IAEA International Agency Energy Agency, 2014.

[13] T. L. Saaty, " How to make a decision: the analytic hierarchy process", European journal of operational research, 48(1), p.9-26, 1990

[14] W. Edwards, "How to use multiattribute utility measurement for social decision making," IEEE Transactions on Systems, Man, and Cybernetics SMC, vol. 7, pp. 326 - 340, 1977.

Content includes material subject to (C) Crown copyright (2017), Dstl. This material is licensed under the terms of the Open Government Licence except where otherwise stated. To view this licence,

visit http://www.nationalarchives.gov.uk/doc/open-government-licence/version/3 or write to the Information Policy Team, The National Archives, Kew, London TW9 4DU, or email:psi@nationalarchives.gsi.gov.uk. 